\title{
EFEITO TEMPORAL SOBRE A RESPOSTA DA PERCEPÇÃO SUBJETIVA DO ESFORÇO
}

\author{
TEMPORAL EFFECT ON THE RESPONSE OF SUBJECTIVE PERCEIVED EXERTION \\ Artigo Original
}

EFECTO TEMPORAL SOBRE LA RESPUESTA DE LA PERCEPCIÓN SUBJETIVA DEL ESFUERZO

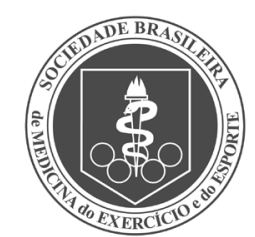

Rafael Evangelista Pedro 1,3

(Profissional de Educação Física)

Ricardo Santos Oliveira'

(Profissional de Educação Física)

Paulo Sérgio de Souza Vasconcelos ${ }^{2}$ (Profissional de Educação Física)

Raymundo Pires Junior ${ }^{2}$

(Profissional de Educação Física)

Vinicius Flavio Milanez ${ }^{1,3}$

(Profissional de Educação Física)

1. Grupo de Estudo das Adaptações Fisiológicas ao Treinamento (Geafit) da Universidade Estadual de Londrina (UEL), Londrina, PR, Brasil. 2. Departamento de Educação Física da Universidade do Norte do Paraná (UNOPAR), Londrina, PR, Brasil.

3. Programa de Pós-graduação Associado em Educação Física Universidade Estadual de Maringál Universidade Estadual de Londrina, PR, Brasil.

\section{Correspondência:}

Vinicius Flavio Milanez

Av. Inglaterra, 1168 apto 14. 86046-000. Londrina, PR, Brasil.

viniciunesp@hotmail.com

\section{RESUMO}

Introdução: A percepção subjetiva de esforço da sessão (PSE da sessão) é um método de fácil aplicação e de muita utilidade no contexto esportivo, sendo sugerido um período de 30 min após a sessão de treinamento para sua avaliação; no entanto, não existem dados empíricos que suportem essa sugestão. Objetivo: Verificar a correlação e a concordância entre a percepção subjetiva de esforço (PSE) média reportada durante sessões de treinamento com valores de PSE reportados 5, 15 e 30 min após o término das sessões de treinamento, em atletas de basquetebol de ambos os gêneros. Métodos: Participaram desse estudo 11 atletas do sexo feminino e nove atletas do sexo masculino. A PSE foi avaliada através da escala de PSE CR-10 a cada 20 min durante a sessão de uma hora de treinamento (PSE média da sessão) e 5, 15 ou 30 min após o término. Dessa forma, a PSE média de cada sessão de treinamento foi comparada com os valores de PSE reportados 5, 15 e 30 min após as sessões. Resultados: Não houve diferença significante da PSE média entre as três sessões. As maiores correlações foram observadas entre PSE média das sessões 2 e 3 com a PSE reportada 15 e 30 min, após para homens $(r=0,92-0,91)$ e mulheres $(r=0,75-0,82)$. Os maiores valores de concordância foram verificados entre a PSE média das sessões 1 e 2 e as reportadas 15 min e 30 min após para ambos gêneros. Conclusão: Com base nos resultados, sugerimos que se evite reportar a PSE 5 min após o término da sessão. Além disso, recomendamos reportar a PSE no mínimo 15 min e, sobretudo, 30 min após o término da sessão.

Palavras-chave: esportes, esforço físico, frequência cardíaca.

\section{ABSTRACT}

Introduction: The session rating perceived exertion (session-RPE) is an easily applied and very useful method in the sports context, a 30 minutes period after training session having been recommended for assessment; however, there are not empiric data supporting this suggestion. Objective: To verify the relationship and the agreement between the average rating of perceived exertion (RPE) during the session with RPE values reported 5, 15 and 30 minutes after the end of the sessions. Methods: Eleven female and nine male basketball players took part at the study. The RPE was assessed by means of a CR-10 scale every 20 minutes during the session which had a duration of $1 \mathrm{~h}$ (average RPE session) and 5, 15 or 30 minutes after de end of the sessions. Thus, the average RPE of every session was compared with RPE values reported 5, 15 and 30 minutes after the respective session. Results: There was no difference in average RPE among the three sessions. The higher correlations were observed between average RPE session during sessions 2 and 3 with RPE reported 15 and 30 minutes after the end of the session for men $(r=0.92-0.91)$ and women $(r=0.75-0.82)$. The higher agreement values were founded between average RPE session during the sessions 1 and 2 with the RPE reported 15 and 30 minutes after the end those sessions for both genders. Conclusions: Based on these results, we suggest avoiding reporting the RPE session 5 minutes after the end of the session. Furthermore, we suggest reporting the RPE at least 15 minutes, or rather 30 minutes after the end of the session.

Keywords: sports, physical exertion, heart rate.

\section{RESUMEN}

Introducción: La percepción subjetiva del esfuerzo de la sesión (PSE de la sesión) es un método de fácil aplicación y de mucha utilidad en el contexto deportivo, siendo sugerido un período de 30 minutos tras la sesión de entrenamiento para su evaluación; sin embargo, no existen datos empíricos que apoyen esta sugerencia. Objetivo: Verificar la correlación y la concordancia entre la percepción subjetiva del esfuerzo (PSE) media reportada a lo largo de las sesiones de entrenamiento con valores de PSE reportados 5, 15 y 30 minutos tras el término de las sesiones de entrenamiento, en atletas de baloncesto de ambos sexos. Métodos: Participaron en este estudio 11 atletas del sexo femenino y 9 atletas del sexo masculino. La PSE fue evaluada por medio de la escala de PSE CR-10 a cada 20 minutos a lo largo de la sesión de una hora de entrenamiento (PSE media de la sesión) y 5, 15 o 30 minutos después del término. De esta forma, la PSE media de cada sesión de entrenamiento fue comparada con los valores de PSE reportados 5, 15 y 30 minutos después de las sesiones. Resultados: No hubo diferencia significativa de la PSE media entre las 3 sesiones. Las mayores correlaciones fueron observadas entre PSE media de las sesiones 2 y 3 con PSE reportada 15 y 30 minutos, después para hombres $(r=0,92-0,91)$ y mujeres $(r=0,75-0,82)$. Los mayores valores de concordancia fueron verificados entre la PSE media de las sesiones 1 y 2 y las reportadas 15 y 30 minutos después para ambos sexos. Conclusión: Con base en los resultados, sugerimos que se evite reportar la PSE 5 minutos tras el término de la sesión. Además, recomendamos reportar la PSE al mínimo 15 minutos e, sobre todo, 30 minutos tras el término de la sesión.

Palabras clave: deportes, esfuerzo físico, frecuencia cardíaca. 


\section{INTRODUÇÃO}

O método percepção subjetiva de esforço (PSE) da sessão tem sido muito utilizado no ambiente esportivo como uma ferramenta útil na quantificação da carga interna de treinamento $(C T)^{1-10}$. Este método surgiu a partir da necessidade de quantificar a CT de maneira prática, rápida e sem gasto operacional extensivo, o que não era possível com os métodos utilizados até o momento, como medidas de frequência cardíaca (FC) e concentração sanguínea de lactato ([La]) devido ao alto custo e a necessidade de avaliadores experientes, além da demora para as análises, o que não possibilita, se necessário, ajustes na CT dos atletas.

Correlações foram encontradas entre as intensidades estimadas a partir da escala de PSE, com medidas objetivas de intensidade tais como FC e [La] em situações de treinamento ${ }^{8,11}$ e competição ${ }^{12}$ na modalidade de karate. Além disso, correlações entre o método PSE da sessão e outros métodos de quantificação de cargas de treinamento baseados na FC e La também foram verificada em muitos esportes como basquetebol ${ }^{5,7}$, futebol masculino ${ }^{6}$, futebol feminino ${ }^{2}$ por rúgbi ${ }^{1}$ e karate $^{10}$, possibilitando sua utilização de forma confiável. Além disso, é sugerido que em alguns casos o método PSE da sessão seja superior aos outros métodos para quantificar a CT, pois a FC pode não responder de forma acurada a todas as ações que são realizadas durante os treinamentos ${ }^{10}$.

Embora a quantificação da CT por meio da PSE da sessão seja fácil, ainda há a necessidade de maiores explicações em relação aos procedimentos para a coleta das informações. É sugerido que a escala de PSE seja apresentada para os atletas somente 30 min após o término da sessão de treinamento, para que exercícios realizados na parte final da sessão de treinamento não tenham maior influência na CT total da sessão, aumentando ou diminuindo sua magnitude ${ }^{5,6}$. Entretanto, de acordo com nosso conhecimento, não há evidências que suportem a utilização de 30 min para que a PSE dos atletas seja quantificada.

Dessa forma, o objetivo do presente estudo foi verificar a correlação e a concordância entre os valores de PSE reportados durante sessões (PSE média da sessão) de treinamento com valores de PSE reportados 5, 15 e 30 min após o término das sessões de treinamento, em atletas de basquetebol de ambos os gêneros. Nossa hipótese é que a avaliação da PSE 5 min após o término da sessão não representará intensidade média da sessão de treinamento; no entanto, acreditamos que não seja necessário aguardar 30 min para que a PSE seja avaliada.

\section{MÉTODOS}

Participaram desse estudo 11 atletas do sexo feminino (Média (DP); idade 15,7 (0,9) anos; massa corporal 68,8 (7,9) kg; estatura $174(5,7) \mathrm{cm}$ e IMC $\left.24,2(2,8) \mathrm{kg} / \mathrm{m}^{2}\right)$ e nove atletas do sexo masculino (idade 16,6 $(0,7)$ anos; massa corporal 70,8 $(10,7) \mathrm{kg}$; estatura $174(5,7) \mathrm{cm}$ e IMC 22,2 $(2,3) \mathrm{kg} / \mathrm{m}^{2}$. Os atletas faziam parte da equipe londrinense de basquetebol, com participação em torneios regionais, estaduais e nacionais. Após receberem informações a respeito dos procedimentos do estudo, os pais ou responsáveis pelos atletas assinaram um termo de consentimento livre e esclarecido. Este estudo foi aprovado pelo Comitê de Ética em Pesquisa envolvendo seres humanos da Universidade do Norte do Estado (Unopar) Londrina, PR, Brasil., protocolado sob o número 0019/2011 em acordo com a resolução 196/96 do Conselho Nacional da Saúde.

Inicialmente os atletas passaram por um processo de familiarização aos procedimentos e ao instrumento utilizado no estudo por um período de quatro semanas. Após o período de familiarização, cada grupo (masculino e feminino) realizou três sessões de treinamento com duração de 60 min cada.

As sessões de treinamento tiveram a mesma duração e as mesmas características para que os valores de PSE médios durante as sessões fossem similares. Cada sessão foi subdividida em três partes de 20 min, para cálculo da PSE média de cada sessão. Na primeira parte os atletas realizaram o aquecimento juntamente com o treinamento técnico. No treinamento técnico os atletas praticaram passes, arremessos de distâncias curtas, médias e longas, e bandejas. Na segunda parte os atletas efetuaram os treinamentos técnicos e táticos tanto para o sistema defensivo quanto para o sistema ofensivo. E na terceira parte os atletas realizaram os coletivos.

Em cada dia de treinamento a PSE foi avaliada em quatro momentos, a cada 20 min durante a sessão de treinamento e 5, 15 ou 30 min após o término da sessão de treinamento. A PSE foi avaliada através da escala de PSECR-10 13 adaptada ${ }^{5}$ e traduzida para língua portuguesa ${ }^{14}$ (tabela 1). Dessa forma, PSE média de cada sessão de treinamento foi comparada com os valores de PSE reportados nos momentos 5, 15 e 30 min após as respectivas sessões (figura 1). As sessões de treinamento foram realizadas em dias diferentes, com um intervalo de 48 h entre cada sessão. A duração do treinamento e o tempo para as coletas da PSE foram verificados com o uso de cronômetro (Timex ${ }^{\circledR}$ ).

Tabela 1. Escala de Borg ${ }^{13}$ adaptada por Foster ${ }^{19}$, traduzida por Gomes do Nascimento $F^{14}$

\begin{tabular}{c|c}
\hline Classificação & Descritor \\
\hline 0 & Repouso \\
\hline 1 & Muito, muito fácil \\
\hline 2 & Fácil \\
\hline 3 & Moderado \\
\hline 4 & Um pouco difícil \\
\hline 5 & Difícil \\
\hline 6 & - \\
\hline 7 & Muito difícil \\
\hline 8 & - \\
\hline 9 & - \\
\hline 10 & Máximo \\
\hline
\end{tabular}

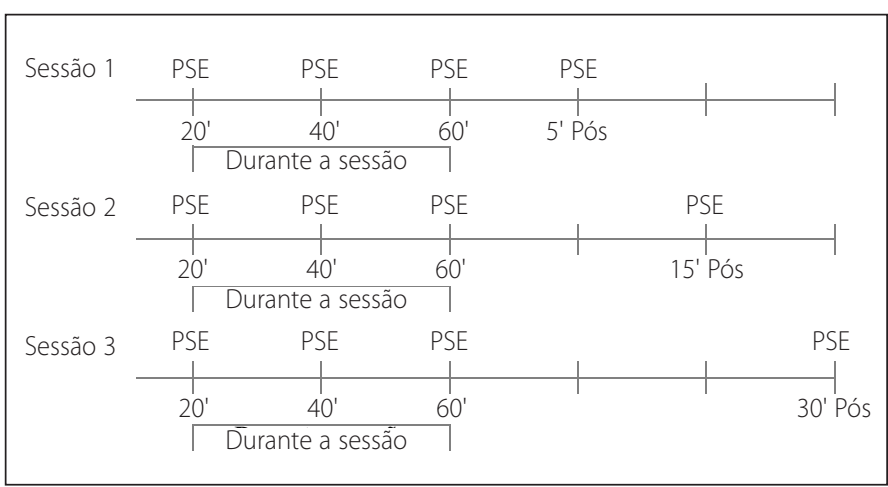

Figura 1. PSE média (média das 3 medidas durante a sessão) reportada durante as sessões 1, 2 e 3, comparadas com a PSE reportada 5, 15 e 30 min após cada sessão respectivamente.

\section{Análise estatística}

A distribuição dos dados foi verificada a partir do teste de Shapiro-Wilk, como os dados não apresentaram distribuição normal, eles foram transformados em logaritmo natural (LN); entretanto, para melhor entendimento, os dados são apresentados no texto e nas ilustrações sem a transformação em LN.

Para comparar a PSE média entre as três sessões de treinamento foi utilizada a análise de variância (ANOVA) para medidas repetidas, com a esfericidade sendo analisada a partir do teste de Mauchly, sendo utilizada a correção de Greenhouse-Geisser quando violado o pressuposto de esfericidade. A PSE média de cada sessão de treinamento (1, 2 e 3) foi comparada respectivamente com a PSE reportada após (5, 15 e 30 min) o término da sessão com o teste $t$ de student pareado.

Para correlacionar a PSE média das sessões (1, 2 e 3) com o valor de PSE reportado após o término da sessão (5, 15 e 30 min) foi utilizado o coeficiente de correlação momento produto de Pearson. A correlação foi avaliada qualitativamente de acordo com as recomendações de Hopkins et al. ${ }^{15}$ : trivial $r<0,1$, pequena $r \geq 0,1$ e $<0,3$, moderada $r \geq 0,3<0,5$, alta $r \geq 0,5$ e $<0,7$, muito alta $r \geq 0,7$ e $<0,9$, quase perfeita $r>0,9$ e perfeita $r=1$. Medidas de concordância entre a PSE média das sessões (1,2 e 3) com as reportadas após as sessões (5, 15 e 30 min) foram analisadas, 
de acordo com os procedimentos descritos na literatura ${ }^{16}$. A significância adotada foi $\mathrm{P}<0,05$. As análises foram realizadas com o programa Statistical Package for the Social Science (SPSS) versão 17.0 para Windows ${ }^{\circledR}$.

\section{RESULTADOS}

Não houve diferença significante ( $F=0,73 ; P=0,44$ e $F=1,74 ; P=0,20)$ entre a PSE média das três sessões de treinamento para homens e mulheres. A PSE média das sessões de treinamento (1, 2 e 3) e após o término de cada sessão (5, 10 e 15 min) para ambos os gêneros estão apresentados na tabela 2.

Houve diferença significativa entre a PSE média da sessão 1 com o valor de PSE reportado 5 min após o término da sessão para os homens e para as mulheres ( $P=0,028 ; P=0,013$, respectivamente); entretanto quando comparada a PSE média das sessões (2 e 3 ) com os tempos de 15 e 30 min respectivamente, não foi observado diferença significante ( $P>0,05)$ em ambos os gêneros (tabela 2).

Os dados referentes às correlações entre a PSE média das sessões com os valores de PSE reportados após as sessões para ambos os gêneros estão apresentados na tabela 3. As correlações entre PSE média da sessão um e a PSE reportada 5 min após a sessão foram consideradas muito alta ( $r=$ $0,76)$ e pequena $(r=0,17)$ para os atletas dos grupos masculino e feminino, respectivamente. Entre a PSE média da sessão 2 e a PSE reportada 15 min após a sessão e entre a PSE média da sessão 3 e a PSE reportada 30 min após a sessão, as correlações foram quase perfeita ( $r=0,92$ e $r=0,91)$ e muito alta $(r=0,75$ e $r=0,82)$ para os grupos masculino e feminino, respectivamente.

A concordância entre as medidas de PSE média das sessões e os valores de PSE reportados 5, 15 e 30 min após o término das sessões foi verificada através da plotagem de Bland-Altmand (figuras 2 e 3). Para os atletas do gênero masculino a intensidade reportada 30 min após a sessão apresentou um viés $(0,41)$ menor do que o viés das análises entre a PSE média das sessões 1 e 2 com os valores de PSE 5 e 15 min após o término da sessão $(0,89$ e 0,52), respectivamente. Na análise realizada com o grupo do gênero feminino o viés também foi menor na análise realizada 30 min após o término da sessão $3(0,29)$ quando comparado as medidas 15 e 5 min após as sessões 2 e $1(0,52$ e 1,09, respectivamente. Além do menor viés analisado 15 e 30 min após as sessões 2 e 3, respectivamente, notou-se também um estreito limite de concordância para essas análises para ambos os grupos.

Tabela 2. Valores médios de intensidade em cada sessão de treinamento e os valores de intensidade após o término de cada sessão (5, 15 e 30 min) para ambos os gêneros. Dados expressos em média e (desvio padrão).

\begin{tabular}{c|c|c|c|c|c|c|c|c}
\hline & \multicolumn{4}{|c|}{ Masculino $(\mathbf{n}=\mathbf{9})$} & \multicolumn{5}{c}{ Feminino ( $=\mathbf{1 1})$} \\
\cline { 2 - 10 } & PSE médio & $\mathbf{5}$ min & $\mathbf{1 5}$ min & $\mathbf{3 0}$ min & PSE & $\mathbf{5}$ min & $\mathbf{1 5}$ min & $\mathbf{3 0}$ min \\
\hline \multirow{2}{*}{ Sessão 1} & 5,44 & 6,33 & - & - & 3,73 & 4,82 & - & - \\
\cline { 2 - 10 } & $(1,54)$ & $(1,41)^{*}$ & - & - & $(0,93)$ & $(0,87)^{*}$ & - & - \\
\hline \multirow{2}{*}{ Sessão 2 } & 5,48 & - & 6,00 & - & 3,21 & - & 3,73 & - \\
\cline { 2 - 9 } & $(2,14)$ & - & $(1,94)$ & - & $(0,96)$ & - & $(1,35)$ & - \\
\hline \multirow{2}{*}{ Sessão 3 } & 5,70 & - & - & 6,11 & 3,45 & - & - & 3,73 \\
\cline { 2 - 9 } & $(1,34)$ & - & - & $(1,27)$ & $(0,76)$ & - & - & $(1,19)$ \\
\hline
\end{tabular}

* Diferença da PSE média da sessão $P<0,05$.

Tabela 3. Correlações entre PSE média das sessões (1, 2 e 3 ) e os valores de PSE reportados 5, 15 e 30 min após o término das sessões.

\begin{tabular}{c|c|c|c|c|c|c}
\hline & \multicolumn{3}{|c|}{ Masculino $(\mathbf{n}=9)$} & \multicolumn{3}{c}{ Feminino $(\mathbf{n}=11)$} \\
\cline { 2 - 7 } & $\mathbf{5}$ min & $\mathbf{1 5}$ min & $\mathbf{3 0}$ min & $\mathbf{5}$ min & $\mathbf{1 5}$ min & 30 min \\
\hline PSE média sessão 1 & $0,76^{*}$ & - & - & 0,17 & - & - \\
\hline PSE média sessão 2 & - & $0,92^{*}$ & - & - & $0,75^{*}$ & - \\
\hline PSE média sessão 3 & - & - & $0,91^{*}$ & - & - & $0,82^{*}$ \\
\hline
\end{tabular}

\section{DISCUSSÃO}

O principal objetivo do estudo foi verificar a correlação e concordância entre PSE média da sessão com a PSE reportada em três diferentes momentos (5, 15 e 30 min) após o término das sessões de treinamento. Correlações de magnitudes muita alta a quase perfeita foram encon-
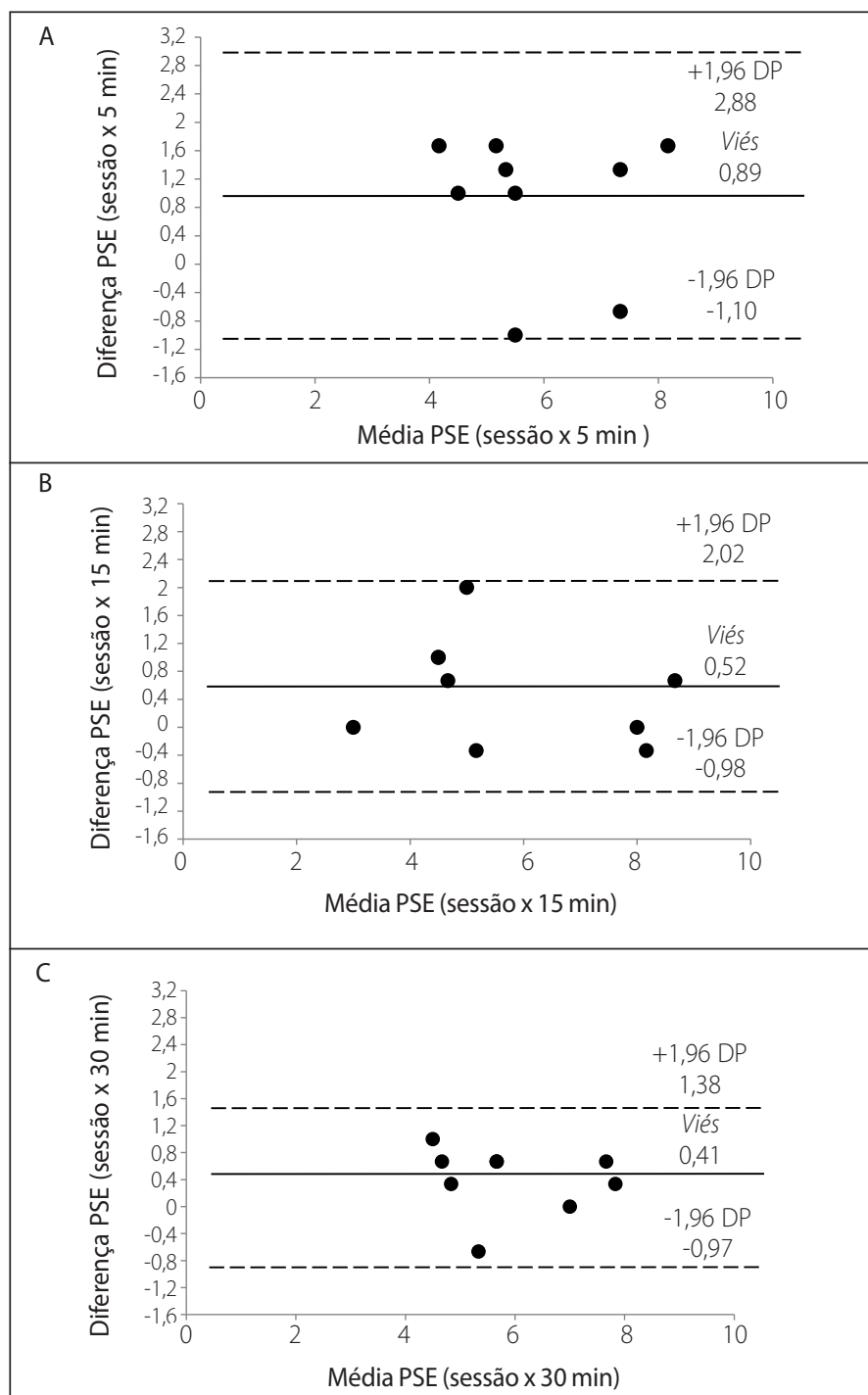

Figura 2. Análise de concordância através da plotagem de Bland-Altman para intensidade do exercício (PSE), verificada durante o exercício e (A) 5; (B) 15 e (C) 30 min após o término da sessão, para homens, $n=9$.

tradas $(0,75$ - 0,92) entre a PSE média das sessões e as PSE reportadas após as sessões para ambos os sexos. No entanto, a correlação entre a PSE média da sessão 1 com a PSE reportada 5 min após a sessão para as atletas do gênero feminino foi pequena $(r=0,17)$. No que diz respeito à concordância, houve baixo viés para as análises entre a PSE média e a PSE reportadas 15 e 30 min após as sessões em ambos os gêneros. O viés foi considerado alto para ambos os sexos quando avaliada a concordância entre a PSE média da sessão e a PSE reportada 5 min após a sessão.

Cautela deve ser adotada ao aplicar a escala de PSE para verificar intensidade após o término da sessão de treinamento conforme sugerido anteriormente ${ }^{5,6}$. Existe a necessidade de se esperar $30 \mathrm{~min}$ após o término da sessão para que os atletas reportem a intensidade da sessão. Segundo alguns autores ${ }^{5,6}$, esse intervalo é necessário para evitar que as atividades realizadas ao final da sessão, sendo de alta ou de baixa intensidade, interfiram desproporcionalmente na resposta dos atletas ${ }^{6}$. Ainda, recomenda-se que o intervalo não seja muito superior a 30 min, uma vez que, períodos muito longos atenuam a avaliação subjetiva da intensidade dos esforços realizados ${ }^{17}$.

Embora essas recomendações apareçam constantemente na literatura, de acordo com o nosso conhecimento, não há estudos que investigaram a influência do tempo após o termino da sessão sobre a resposta da PSE em esportes coletivos, sugerindo que essa recomendação não tenha sido devidamente testada. Consequentemente os resultados da presente pesquisa podem ser considerados como um primeiro passo no que diz 


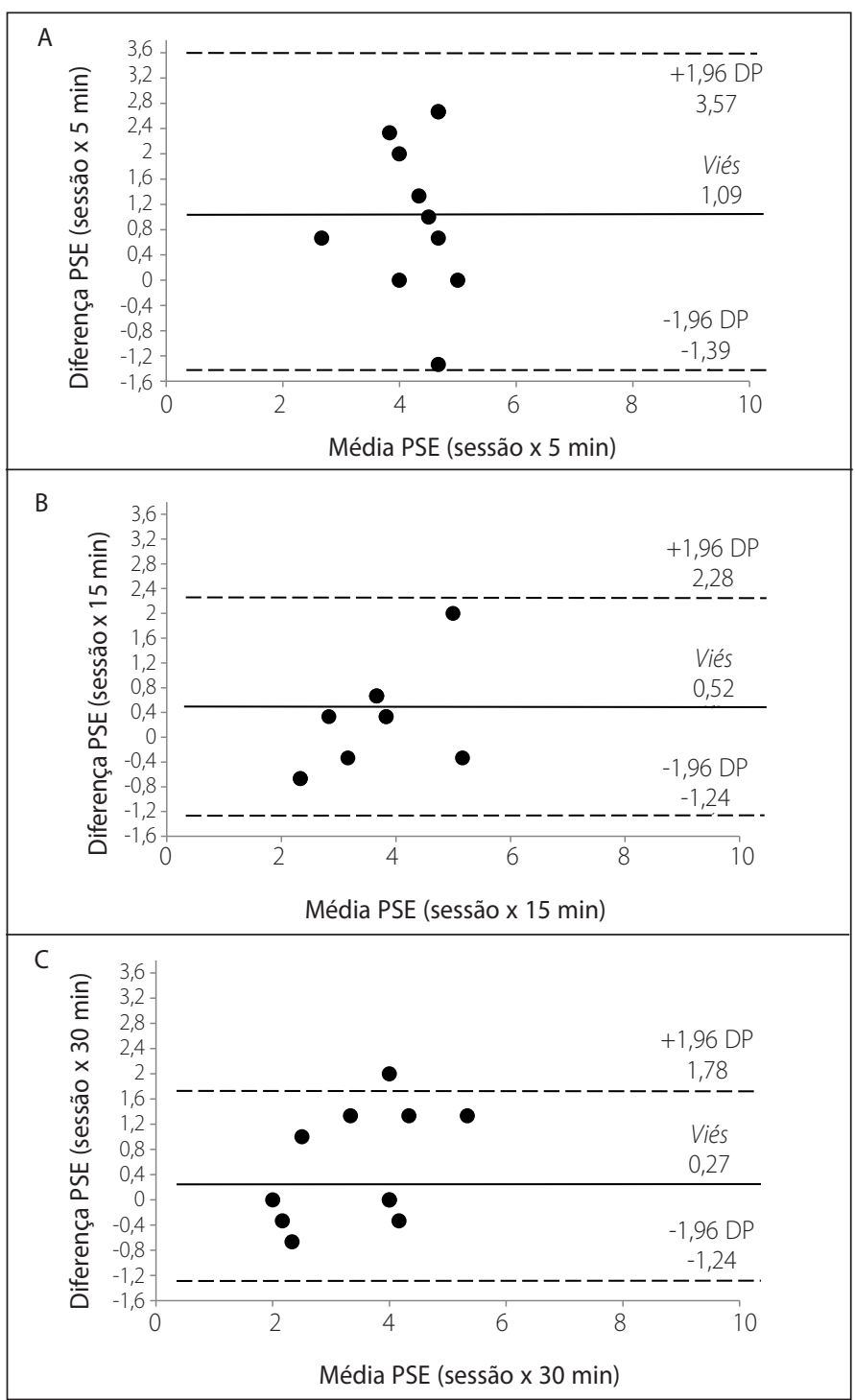

Figura 3. Análise de concordância através da plotagem de Bland-Altman para intensidade do exercício (PSE), verificada durante o exercício e (A) 5, (B)15 e (C) 30 min após o término da sessão, para mulheres, $n=11$.

respeito a essa temática. Correlação de magnitudes muita alta a quase perfeita foram encontradas $(0,7-0,92)$ entre a PSE média das sessões e a PSE reportada após as sessões para ambos os sexos (tabela 1), exceto para a correlação entre a PSE média da sessão 1 com a PSE reportada 5 min após o término da mesma para as atletas do sexo feminino (tabela 2). De acordo com os resultados apresentados no presente estudo é possível afirmar que a PSE reportada a partir de 15 min após a sessão representa

\section{REFERÊNCIAS}

1. Coutts AJ, Murphy A, Pine M, Reaburn P, Impellizzeri F. Validity of the session-RPE method for determining training load in team sport athletes. J Sports Med Sci. 2003;30(1):1042-7.

2. Alexiou $\mathrm{H}$, Coutts AJ. A comparison of methods used for quantifying internal training load in women soccer players. Int J Sports Physiol Perform. 2008;3(3):320-30

3. Borresen J, Lambert MI. Quantifying training load: a comparison of subjective and objective methods. Int J Sports Physiol Perform. 2008;3(1):16-30.

4. Day ML, Mcguigan MR, Brice G, Foster C, Monitoring exercise intensity during resistance training using the session-RPE scale. J Strength Cond Res. 2004;18:353-8.

5. Foster, C, Florhaug, JA, Franklin, J, Gottschall, L, Hrovatin, LA, Parker, S, et al. A new approach to monitoring exercise training. J Strength Cond Res. 2001;15(1):109-15.

6. Impellizzeri FM, Rampinini E, Coutts AJ, Sassi A, Marcora SM. Use of RPE-based training load in soccer. Med Sci Sports Exerc. 2004;36(6):1042-7.

7. Manzi V, D'ottavio S, Impellizzeri FM, Chaouachi A, Chamari K, Castagna C. Profile of weekly training load in elite male professional basketball players. J Strength Cond Res. 2010;24(5):1399-406.

8. MilanezVF, Spiguel-Lima MC, Gobbato CA, Perandini LA, Nakamura FY, Ribeiro LF. Correlates of session-rate of perceived exertion (RPE) in a karate training session. Sci Sports. 2010;26:38-43.

9. Milanez V, Pedro R, Moreira A, Boullosa D, Salle-Neto F, Nakamura F. The role of aerobic fitness on session-rating of perceived exertion in futsal players. Int I Sports Physiol Perform. 2011;6:358-66.

10. MilanezVF, Pedro RE. Aplicação de diferentes métodos de quantificação de cargas durante uma sessão a PSE média das sessões de treinamento devido à alta correlação e a alta concordância encontrada para essas análises.

Entretanto, nossos resultados trazem evidências de que essa sugestão realmente deva ser seguida, visto que a análise de concordância para os dados de PSE médio da sessão 1 e os valores de PSE reportados 5 min após o término da sessão apresentou um valor de viés elevado para os homens $(0,89)$ e mulheres $(1,09)$. O risco de viés foi menor entre a PSE média da sessão e PSE reportada 15 min após a sessão para ambos os sexos $(0,52)$ e ainda menor entre a PSE média da sessão e PSE reportada 30 min após a sessão tanto para os homens $(0,41)$, quanto para as mulheres $(0,27)$. Diferente dos resultados apresentados no presente estudo, Kilpatrick et al. ${ }^{18}$ em um delineamento laboratorial, utilizando a escala de percepção de esforço Omini, não encontraram diferenças significantes entre as medidas de intensidade imediatamente e 15 min após 30 min de exercícios em ciclo ergômetro com três diferentes intensidades (leve, moderada e vigorosa).

As divergências entre os resultados do presente estudo e o estudo proposto por Kilpatrick et al. ${ }^{18}$ podem ser pelo fato de os autores terem utilizado um exercício contínuo, o que difere da natureza das sessões de treinamento utilizadas no presente estudo, além de os exercícios terem sido realizados em condições laboratoriais. As outras possíveis limitações do estudo de Kilpatrick et al. ${ }^{18}$ podem ter sido causadas pelo fato dos autores não terem comparado os valores de PSE após o exercício com os valores médios do exercício para ter conhecimento se esses valores seriam equivalentes. Além desse ponto, existe a análise estatística que pode conter alguns erros, sendo que os próprios autores sugerem mais estudos fora do ambiente laboratorial.

Dessa forma, os resultados do presente estudo confirmam as recomendações empíricas da necessidade de se respeitar o período mínimo de 30 min para evitar que as atividades realizadas ao final da sessão interfira desproporcionalmente na resposta dos atletas. No entanto, se não for possível aguardar o término do intervalo citado, considera-se a espera de no mínimo 15 min após a sessão de treinamento para avaliar a PSE dos atletas durante as sessões de treinamento. Entretanto sugerimos a realização de mais estudos com aleatorização dos avaliados e das sessões, bem como, aumento do número de sessões, uma vez que, essas foram limitações do presente estudo.

\section{CONCLUSÃO}

De acordo com os resultados do presente estudo, deve-se evitar reportar PSE 5 min após a sessão de treinamento devido à falta de concordância com a PSE média das sessões. Consequentemente, para uma melhor concordância de resultados com o valor médio da sessão, recomenda-se reportar a PSE no mínimo 15 e ou, sobretudo 30 min após o término da mesma.

Todos os autores declararam não haver qualquer potencial conflito de interesses referente a este artigo.

de treinamento de karate. Rev Bras Med Esporte. 2012;18(4):278-82

11. Milanez VF, Lima MCS, Gobbato CA, Nakamura FY, Cyrino ES. Avaliação e comparação das respostasdo esforço percebido e concentração de lactato durante uma sessão de treinamento de caratê. Rev Educ Fis UEM. 2009;20(4):607-13.

12. Milanez VF, Lima MCS, Perandini L, Gonçalves C, Franchini E. Avaliação e comparação das respostas da percepção subjetiva do esforço e concentração de lactato em uma competição oficial de karate. Rev Educ Fis UEM. 2011;22(1):57-64.

13. Borg GA. Psychophysical bases of perceived exertion. Med Sci Sports Exerc. 1982;14(5):377-81.

14. Gomes do Nascimento F. Escalas de Borg para a dor e o esfoço percebido. São Paulo: Manole; 2000.

15. Hopkins W. A scale of magnitudes for effect. Available from URL: statistics.http://www.sportsci.org/ resource/stats/index.html. [Accessed March 31, 2012].

16. Bland JM, Altman DG. Statistical methods for assessing agreement between two methods for clinical measurement. Lancet. 1986;1 (8476):307-10.

17. Nakamura F, Moreira A, Aoki MS. Monitoramento da carga de treinamento: a percepção subjetiva do esforço da sessão é um método confiável? Rev Educ Fis UEM. 2010;21(1):1-11.

18. Kilpatrick MW, Robertson RJ, Powers JM, Mears LL, Ferrer NF. Comparisons of RPE before, during, and after self-regulated aerobic exercise. Med Sci Sports Exerc. 2009;41(3):682-7.

19. Foster C. Monitoring training in athletes with reference to overtraining syndrome. Med Sci Sports Exerc. 1998;30(7):1164-8. 